\title{
Great Geomagnetic Storms in the Rise and Maximum of Solar Cycle 23
}

\author{
A. Dal Lago ${ }^{1,2}$, L. E. A. Vieira ${ }^{1,2}$, E. Echer ${ }^{1}$, W. D. Gonzalez ${ }^{1}$, A. L. Clúa de Gonzalez ${ }^{1}$, \\ F. L. Guarnieri ${ }^{1}$, L. Balmaceda ${ }^{1}$, J. Santos ${ }^{1}$, M. R. da Silva ${ }^{1}$, A. de Lucas ${ }^{1}$, and N. J. Schuch ${ }^{3}$ \\ ${ }^{1}$ Fundação de Amparo à Pesquisa do Estado de São Paulo, FAPESP, SP, Brazil \\ ${ }^{2}$ Instituto Nacional de Pesquisas Espaciais, São José dos Campos, SP, Brazil \\ ${ }^{3}$ Centro Regional Sul de Pesquisas Espaciais, Santa Maria, RS, Brazil
}

Received on 21 January, 2004; revised version received on 18 May, 2004

\begin{abstract}
Geomagnetic storms are intervals of time when a sufficiently intense and long-lasting interplanetary convection electric field leads, through a substantial injection of energy into the magnetosphere-ionosphere system, to an intensified ring current, strong enough to exceed some key threshold of the quantifying storm time Dst index. We have studied all the 9 great magnetic storms (peak Dst $<-200 \mathrm{nT}$ ) observed during the rise and maximum of solar cycle 23 (from 1997 to early 2001), in order to identify their solar and interplanetary causes. Apart of one storm occurred during the period without observations from the Solar and Heliospheric Observatory (SOHO), all of them were related to coronal mass ejections observed by the Large Angle and Spectroscopic Coronagraph (LASCO). The sources of interplanetary southward magnetic field, Bs, responsible for the occurrence of the storms were related to the intensified shock/sheath field, interplanetary magnetic cloud's field, or the combination of sheath-cloud or sheath-ejecta field. It called our attention the fact that one of the events was related to a slow CME, with CME expansion speed not greater than $550 \mathrm{~km} / \mathrm{s}$. The purpose of this paper is to address the main sources of large geomagnetic disturbances using the current satellite capability available. As a general conclusion, we found that shock/sheath compressed fields are the most important interplanetary causes of great magnetic storms during this period.
\end{abstract}

\section{Introduction}

On one hand we have the solar wind plasma constantly flowing out of the Sun throughout interplanetary space at typical speeds of the order of $400-500 \mathrm{~km} / \mathrm{s}$, carrying the Sun's magnetic field frozen into it, given its high conductivity [1]. Superposed on this ambient plasma there are transient injections of material, often faster than the solar wind, and also carrying strong magnetic field, the so called coronal mass ejections [2]. To complete the set of structures present in the interplanetary medium, we can find the high speed solar wind streams, which have lower density than the solar wind, and travel with speeds of the order of $800 \mathrm{~km} / \mathrm{s}$, coming from the coronal holes [3]. On the other hand, we have the Earth's magnetic field, shielding Earth from the variety of interplanetary structures mentioned above, forming the cavity known as magnetosphere [4]. If the solar wind magnetic field is such that its direction points anti-parallel to the Earth's magnetic field, energy can be injected into the magnetosphere, increasing the equatorial ring current, causing a geomagnetic storm [5]. Gonzalez and Tsurutani [6] found that a dawn-dusk convection electric field greater than $5 \mathrm{mV} / \mathrm{m}$, which means $10 \mathrm{nT}$ magnetic field and $420 \mathrm{~km} / \mathrm{s}$ speed, lasting for at least 3 hours, is the minimum interplanetary condition for the occurrence of an intense geomagnetic storm, i.e. storm-time Dst index less than -100 nT. Tsurutani et al. [7] studied the 5 great geomagnetic storms, with Dst $<-250 \mathrm{nT}$, observed in the period of 1978-1979 and they found two types of interplanetary cause for these storms, one being shock compressed magnetic field ( 3 events), and the other being ejecta/magnetic cloud field ( 2 events). The first type is a shock wave propagating in the solar wind, probably driven by a CME related structure (ejecta), which compresses the existing solar wind magnetic field, that by chance can be pointing anti-parallel to the Earth's magnetic field. If we consider a frame with x-direction in the Sun-Earth line, pointing to the Sun, and the z-direction pointing along the Earth's magnetic field in the equatorial region, the interplanetary magnetic field capable of causing a geomagnetic storm should be pointing in the negative z-direction, or southward direction (Bs), which is the Earth's magnetic field anti parallel direction. The second type of interplanetary structure responsible for the storms, also called geoeffective structures, found by Tsurutani et al. [7] were interplanetary ejecta, which are believed to be the ejected material from the CMEs observed by coronagraphs, near the Sun. These ejecta can be very well behaved magnetic structures, like interplanetary magnetic clouds [8], modeled by a force-free $(\vec{J} \times \vec{B}=0)$ magnetic flux rope assuming local cylindrical symmetry [9]. Very often, magnetic clouds possess intense magnetic field, with a substantial fraction pointing southward. Another source of very intense geomagnetic storms is the resulting magnetic field from compression of a CME-ejecta by a high speed stream solar wind overtaking it 

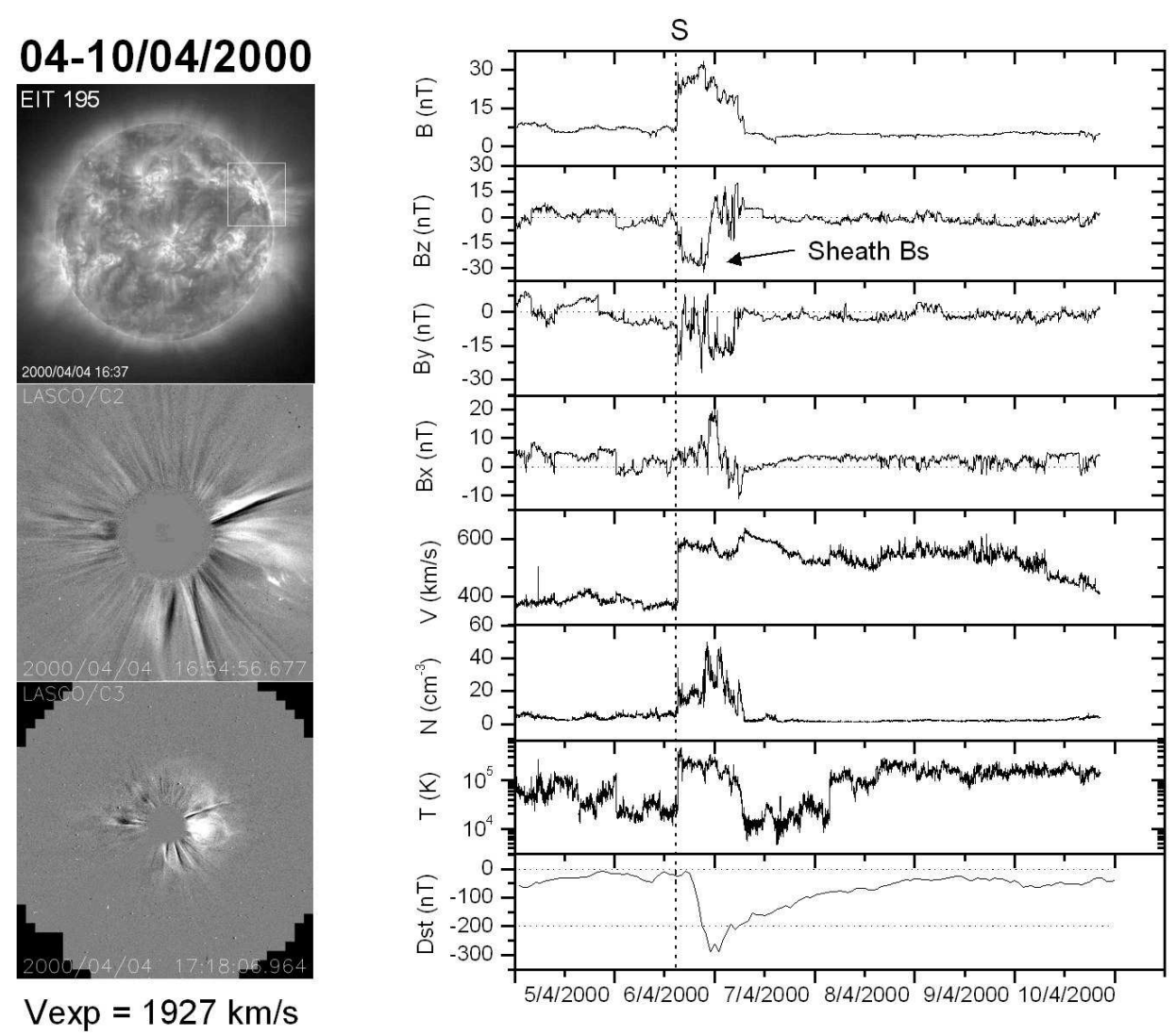

Figure 1. On the left side, from top to bottom: EIT 195 snapshot taken on April 4th (2000), at 16:37 UT showing the flare inside the rectangle; LASCO C2 and LASCO C3 snapshots taken on the same day taken at 16:54 UT and 17:18 UT showing the full halo CME. On the right side, from top to bottom: interplanetary magnetic field and its 3 components, Bz, By and Bx, solar wind (proton) speed, number density and temperature, and the Dst index for the period of April 5th to April 10th, 2000.

[10-12]. Dal Lago et al. [13] have shown that such mechanism can double the geoeffectiveness of an interplanetary ejecta. In this work we shall study the solar and interplanetary causes of the 9 great geomagnetic storms observed from January 1997 to April 2001. We use observations made by the Large Angle and Spectroscopic Coronagraph (LASCO), and by the Extreme Ultraviolet Imaging Telescope (EIT), both on board of the Solar and Heliospheric Observatory (SOHO), able to image the solar corona in white light from 2 to 32 solar radii [14], combined with interplanetary magnetic field and plasma observations made by the Advanced Composition Explorer (ACE) satellite, sitting in the L1 point, close to the Earth. By great storms, we consider those for which the peak of the Dst index was less than $-200 \mathrm{nT}$. In the following sections we will present some of these events, illustrating the various types of solar, interplanetary and geomagnetic characteristics.

\section{April 6-7 (2000) storm}

Figure 1 is a composition of solar, interplanetary and geomagnetic observations from the 4th to the 10th of April
(2000), showing images from EIT and LASCO instruments on the left side, and the magnetic field and plasma parameters observed by ACE, together with the Dst index on the right side. Interplanetary and geomagnetic parameters in Fig. 1 are presented in universal time (UT). Soon after the shock "S", the z-component of the magnetic field turns southward and is intensified because of a compression of the sheath region, remaining like that for approximately 18 hours, making the Dst index to fall to $-321 \mathrm{nT}$. The total magnetic field jumps across the shock from 7 to $25 \mathrm{nT}$. No ejecta structure is observed after the shock, indicating that perhaps the satellite didn't intercept the middle of the structure. This hypothesis is consistent with the solar observations of this event, shown in the left side of Figure 1, where one can see a flare on April 4th at heliographic position N16W66 (top left), thus far from the central meridian. Associated with this flare was a fast CME, with expansion speed of around $1927 \mathrm{~km} / \mathrm{s}$, spreading all around the solar disk, creating a full halo (middle and bottom left). The CME expansion speed is the CME growth rate in the direction approximately perpendicular to the fastest plane-of sky radial CME speed [15]. Dal Lago et al. [16] have shown that this speed is related to the radial CME speed. 


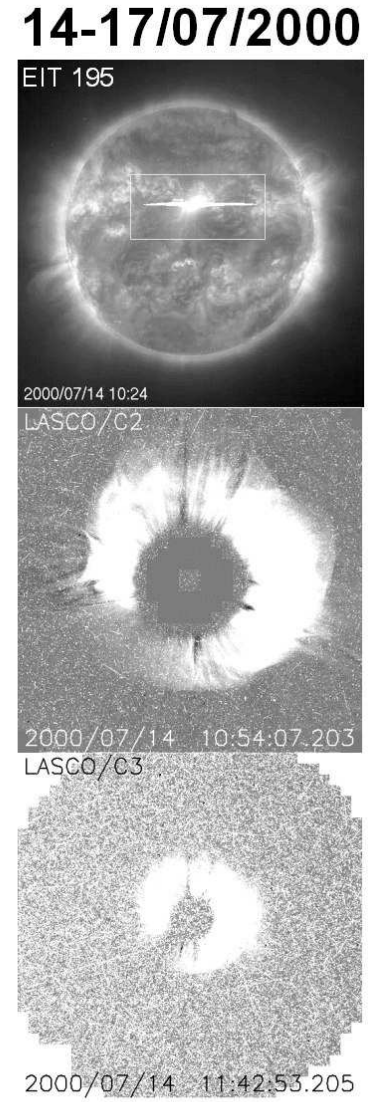

Vexp $=2177 \mathrm{~km} / \mathrm{s}$

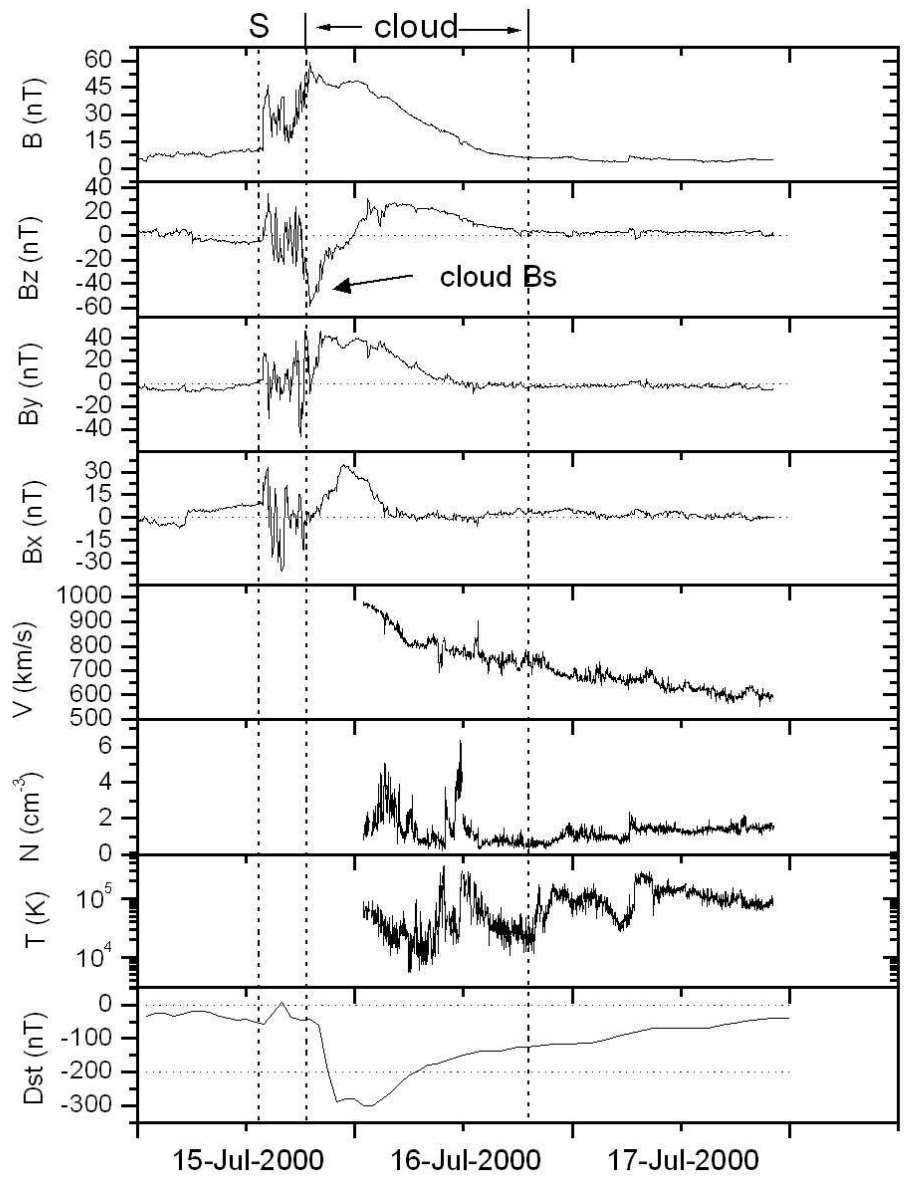

Figure 2. On the left side, from top to bottom: EIT 195 snapshot taken on July 14th (2000), at 10:24 UT showing the flare inside the rectangle; LASCO C2 and LASCO C3 snapshots taken on the same day taken at 10:54 UT and 11:42 UT showing the full halo CME. On the right side, from top to bottom: interplanetary magnetic field and its 3 components, Bz, By and Bx, solar wind (proton) speed, number density and temperature, and the Dst index for the period of July 15th to July 17th, 2000.

\section{July 15-16 (2000) storm}

Figure 2 is the same as Figure 1 but for the period of 1417 of July, 2000, and it shows the very well know "Bastilla event". It consists of an interplanetary shock driven by a magnetic cloud, whose intense magnetic field ( $50 \mathrm{nT}$ ) rotates from south to north smoothly. While pointing southward, it causes a very intense fall in the Dst index, reaching its minimum of $-300 \mathrm{nT}$. EIT showed that a flare occurred on July 14th, at central meridian, heliographic position N17E01, from AR9077 (Figure 2, top left). The interplanetary shock at 1 AU indicated by the " $S$ " line in Fig. 2 could only be identified in the magnetic field data, because the plasma detector aboard ACE suffered a temporary black-out as a consequence of the flare accelerated particles. Associated with this flare was a full halo CME, observed by the LASCO instrument, with an expansion speed of $2177 \mathrm{~km} / \mathrm{s}$, shown on the same figure on the middle and bottom left panels.

\section{October 22nd (1999) storm}

Figure 3 shows the same kind of composition of the previous figures, but for the period of 17-23 of October (1999). Again, on the right side of the figure we have magnetic field and plasma parameters observed by ACE, together with the Dst index. The dotted line " $S$ " indicates the arrival of a shock, followed by an ejecta, which was not of a magnetic cloud type. The average magnetic field of the estructure is of the order of $20 \mathrm{nT}$, except at the rear part, where it jumps to around $36 \mathrm{nT}$. At this rear portion of the ejecta, the magnetic field was pointing substantially southward, thus causing the Dst to fall to $-237 \mathrm{nT}$. Following this ejecta, one can observe a high speed stream, which is overtaking it. At the interface, a jump in speed, density and temperature is present. Probably this interaction was the cause of the magnetic field of the ejecta to jump, as a result of compression. This event is very interesting, because the related CME observed by LASCO (middle and bottom panels of Figure 3 ) had an expansion 
speed of $546 \mathrm{~km} / \mathrm{s}$, which is considered a slow speed, very close to the normal solar wind speed. Also, this CME was not very much traveling towards Earth because it was a partial halo CME, i.e. its main propagation direction was was a partial halo $\mathrm{CME}$, i.e. its main propagation direction was considerably out of the Sun-Earth direction. Nevertheless, it caused a very intense geomagnetic storm. This event is unique in the group, and our conclusion is that the cause of the storm was mostly interplanetary interactions, rather than solar.
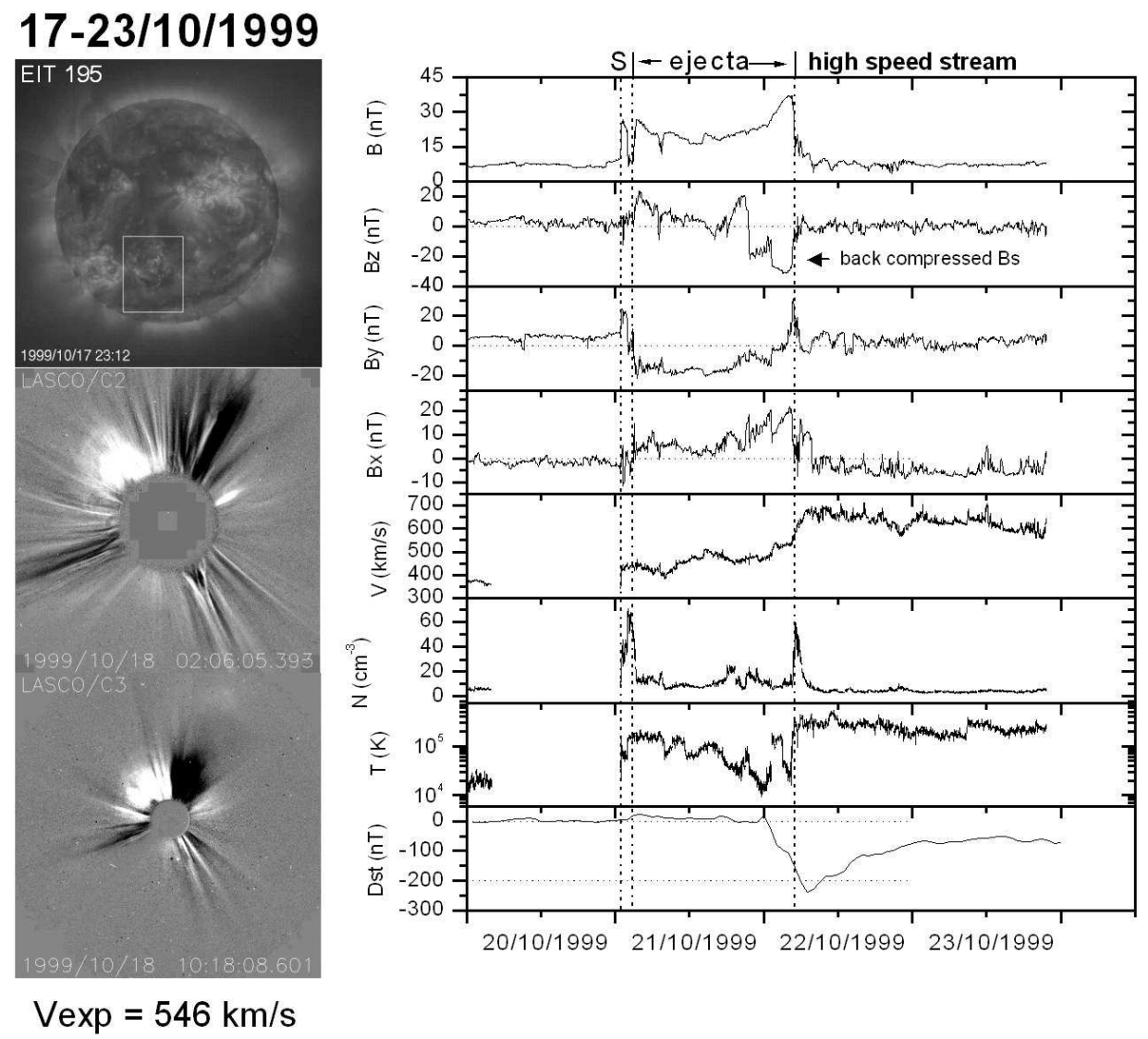

Figure 3. On the left side, from top to bottom: EIT 195 snapshot taken on October 17th (1999), at 23:12 UT showing the flare inside the rectangle; LASCO C2 and LASCO C3 snapshots taken on October 18th (1999) at 02:06 UT and 10:18 UT showing the partial halo CME. On the right side, from top to bottom: interplanetary magnetic field and its 3 components, Bz, By and Bx, solar wind (proton) speed, number density and temperature, and the Dst index for the period of October 20th to October 23th, 1999.

\section{Summary and conclusions}

Table I gives information about the 9 events considered in this paper, indicating their solar and interplanetary characteristics, as well as the corresponding geomagnetic effect. For each event, peak Dst values as well as date and time of their occurrences are indicated in the "Dst" and "Dst date/time (UT)" columns, respectively. The solar origins of these events were related to CMEs at the Sun in all cases, with the exception of the September 24-25th (1998) event for which there were no SOHO observations. Out of 8 CMEs, 7 were full halos, spanning all around the solar disk, indicated by the "360" in the "Ext" column, which means these CMEs had substantially component of their main propagation direction lying on the Sun-Earth line. Only one CME was partial, the one observed on October 18th (1999). Observing the CME expansion speeds measured in the LASCO ima- ges and shown in the "Vexp" columns, 6 were greater than $900 \mathrm{~km} / \mathrm{s}$, from which 5 were greater than $1200 \mathrm{~km} / \mathrm{s}$. The October 18th (1999) CME had an expansion speed of 546 $\mathrm{km} / \mathrm{s}$, which is much slower than the others and very close to the normal solar wind speed. One halo CME was extremely faint in the LASCO images, not enabling the measurement of the expansion speed. The heliographic positions of the associated flares observed in EIT images are shown in the column "AR". All but one were within \pm 40 degrees of longitude, close to the central meridian. The exception was the April 4th (2000) CME. The travel time from the Sun to the Earth was, with 2 exceptions, approximately 2 days (50 hours), being one of the exceptions 53 hours and the other 73 hours. The latter one was the slow partial halo CME observed on October 18th (1999). Concerning the interplanetary structures we found that 5 out the 9 events were interplanetary magnetic clouds, as defined by Burlaga et al. 
TABLE I. Solar, interplanetary and geomagnetic parameters form the 9 great geomagnetic storms occurred in the period of January 1997 to April 2001.

\begin{tabular}{|c|c|c|c|c|c|c|c|c|c|c|c|}
\hline CME date/time (UT) & Ext & $V \exp (\mathrm{km} / \mathrm{s})$ & AR & Assoc. & Shock date/time (UT) & $\mathrm{T}$ & Vpr & IP kind & Bs source & Dst & Dst date/time (UT) \\
\hline 02/05/1998 14:06 & 360 & 1228 & S15W15 & 1 & 04/05/1998 02:10 & 36 & 1152 & neje & sheath Bs & -216 & 04/05/1998 06:00 \\
\hline no soho & & & & 2 & 24/09/1998 23:15 & & & cloud & sheath Bs + cloud Bs & -207 & 25/09/1998 10:00 \\
\hline 18/10/1999 00:06 & 210 & 546 & S20E10 & 1 & 21/10/1999 01:34 & 73 & 566 & eje & back compress eje Bs & -237 & 22/10/1999 07:00 \\
\hline 04/04/2000 16:32 & 360 & 1927 & N16W66 & 1 & 06/04/2000 16:02 & 47 & 875 & neje & sheath Bs & -321 & 07/04/2000 01:00 \\
\hline 14/07/2000 10:54 & 360 & 2178 & N17E01 & 1 & 15/07/2000 14:18 & 27 & 1517 & cloud & cloud Bs & -300 & $15 / 07 / 200022: 00$ \\
\hline 09/08/2000 16:30 & 360 & 898 & $\mathrm{~N} 22 \mathrm{E} 12$ & 1 & $11 / 08 / 200018: 19$ & 50 & 834 & cloud & sheath Bs + cloud Bs & -237 & 12/08/2000 10:00 \\
\hline 15/09/2000 11:50 & 360 & $\mathrm{nv}^{*}$ & N13E08 & 2 & $17 / 09 / 200017: 00(?)^{* *}$ & 53 & 782 & cloud & sheath Bs & -201 & $17 / 09 / 2000$ 24:00 \\
\hline 29/03/2001 10:26 & 360 & 1511 & $\mathrm{~N} 20 \mathrm{~W} 19$ & 1 & $31 / 03 / 200100: 14$ & 38 & 1099 & eje & sheath Bs + eje Bs & -377 & 31/03/2001 09:00 \\
\hline 10/04/2001 05:30 & 360 & 2679 & S23W09 & 2 & $11 / 04 / 200115: 18$ & 34 & 1229 & cloud & sheath Bs + cloud Bs & -251 & 12/04/2001 00:00 \\
\hline
\end{tabular}

* CME was not visible enough to enable the expansion speed measurement.

** not a clear shock, lack of discontinuity in the parameters.

Symbols are: 1 = unequivocal association between a CME and an interplanetary event; $2=$ possible association between a CME and an interplanetary event; neje = not an interplanetary ejecta structure of any kind; eje = definitely an interplanetary ejecta but not defined; cloud $=$ interplanetary ejecta of the magnetic cloud type according to Burlaga et al. [8].

as defined by Burlaga et al. [8], indicated in the column "IP Kind" as "cloud", 2 were interplanetary ejecta without the signature of magnetic cloud, indicated by "eje" in this same column, and the other 2 were only compressed sheath regions, as a consequence of an interplanetary shock passage, indicated by "neje". The column "Bs source" indicates the various sources of southward interplanetary magnetic field responsible for the occurrence of each storm. Four sources were found, as follow: (1) shock sheath compressed field in 3 events; (2) shock sheath compressed field combined with internal ejecta field in 4 events; (3) internal ejecta field alone in 1 event; (4) internal ejecta magnetic field compressed by a high speed stream solar wind following the ejecta in 1 event. Thus, our main conclusion is that sheath compressed fields, caused by interplanetary shocks, are present in 7 out of 9 great magnetic storms, and are the most important interplanetary source of these very intense storms. This conclusion was similar to the one obtained by Tsurutani et al. [7] when analyzing the great magnetic storms observed in the period of 1978-1979. Watari et al. [17] also reached a similar conclusion, but for all intense geomagnetic storms (peak Dst $<-100 \mathrm{nT}$ ). When comparing our results with Tsurutani et al. [7], the most significant difference is the October 1823th (1999) event, because it was caused by an interaction between an ejecta and a high speed stream following it.

\section{Acknowledgements}

The authors would like to acknowledge Fundação de Amparo a Pesquisa do Estado de São Paulo (FAPESP) of Brazil for supporting A. Dal Lago and L. E. A. Vieira under the projects $02 / 14150-0$ and $02 / 12723-2$. The authors would also like to acknowledge SOHO/EIT and SOHO/LASCO work teams for making the data available, N. Ness (Bartol Research Institute) for providing ACE magnetic field data, D. J. McComas (SWRI) for providing ACE plasma data, both through CDAWeb, and WDC-Kyoto for providing the Dst index. SOHO is an international cooperation between NASA and ESA.

\section{References}

[1] J. C. Brandt, Introduction to the solar wind. San Francisco (1970).
[2] A. J. Hunduhausen, An introduction. Coronal mass ejections, Washington, DC (1997).

[3] R. Schwenn, Large-scale structure of the interplanetary medium, Chapter 1, Physics of the Inner Heliosphere, SpringerVerlag, Berlin-Heidelberg(1990).

[4] M. G. Kivelson, and C. T. Russell, Introduction to Space Physics, Cambridge University Press, New York (1995).

[5] W. D. Gonzalez, J. A. Joselyn, Y.Kamide, H. W. Kroehl, G. Rostoker, B. T. Tsurutani, and V. M. Vasyliunas, J. Geophys. Res. 99, 5771 (1994).

[6] W. D. Gonzalez, B. T. Tsurutani, Planet. Space Sci. 35, 1101 (1987).

[7] B. T. Tsurutani, W. D. Gonzalez, F. Tang, and Y. T. Lee, Geophys. Res. Lett. 19, 73 (1992).

[8] L. F. Burlaga, E. Sittler, F. Mariani, and R. Schwenn, J. Geophys. Res. 86, 6673 (1981).

[9] H. Goldstein, On the field configuration in magnetic clouds. In Solar Wind Five, NASA Conf. Publ. 2280, Washington DC(1983).

[10] L. F. Burlaga, K. W. Behannon, and L. W. Klein, J. Geophys. Res. 92, 5725 (1987).

[11] V. Bothmer, and R. Schwenn, J. Geomag. Geoelectr. 47, 1127 (1995).

[12] A. Dal Lago, W. D. Gonzalez, A. L. Clua de Gonzalez, and L. E. Vieira, J. Atm. Solar Terr. Phys. 63, 451 (2001).

[13] A. Dal Lago, W. D. Gonzalez, A. L. C. de Gonzalez, and L. E. Vieira, Adv. Space Res. 30, 2225 (2002).

[14] G. E. Brueckner, R. A. Howard, M. J. Koomen, C. M. Korendyke, D. J. Michels, J. D. Moses, D. G. Socker, K. P. Dere, P. L. Lamy, A. Llebaria, M. V. Bout, G. M. Simnett, D. K. Bedford, and C. J. Eyles, Solar Phys., 162, 357 (1995).

[15] R. Schwenn, A. Dal Lago, W. D. Gonzalez, E. Huttunen, C. O. St. Cyr, and S. P. Plunkett, A tool for improved space weather predictions: the CME expansion speed, Eos Trans. AGU, 82, 47, Fall Meet. Suppl., Abstract SH12A0739(2001).

[16] A. Dal Lago, R. Schwenn, and W. D. Gonzalez, Adv. Space Res. 32, 2637 (2003).

[17] S. Watari, M. Vandas, and T. Watanabe, Ann. Geophys. 22, 673 (2004). 